\title{
Key characteristics of walking correlate with bone density in individuals with chronic stroke
}

\author{
Lise C. Worthen, MS; ${ }^{1}$ C. Maria Kim, MSc, PT; ${ }^{1}$ Steven A. Kautz, PhD $;^{2-4}$ Henry L. Lew, MD, PhD; ${ }^{5}$ \\ B. Jenny Kiratli, PhD; ${ }^{6}$ Gary S. Beaupre, $\mathbf{P h D}^{\mathbf{1}^{*}}$ \\ ${ }^{1}$ Department of Veterans Affairs (VA) Bone and Joint Center, VA Palo Alto Health Care System, Palo Alto, CA; ${ }^{2}$ Brain \\ Rehabilitation Research Center, Malcom Randall VA Medical Center, Gainesville, FL; ${ }^{3}$ Department of Physical Therapy, \\ University of Florida (UF), Gainesville, FL; ${ }^{4}$ UF Brooks Center for Rehabilitation Studies, Gainesville, FL; ${ }^{5}$ Spinal \\ Cord Injury Center, VA Palo Alto Health Care System, Palo Alto, CA; ${ }^{6}$ Physical Medicine and Rehabilitation Service, \\ VA Palo Alto Health Care System, Palo Alto, CA
}

\begin{abstract}
Several recent studies of ambulatory stroke survivors have shown decreased bone mineral density (BMD) in the lower limbs and an elevated risk of hip fracture. Because bone mass is linked to skeletal loading, weight-bearing activities of daily living such as walking are considered critically important for maintenance of femoral BMD in ambulatory individuals. Little is known about the relationships between walking characteristics, skeletal loading, and bone maintenance in individuals who have experienced a stroke. This study determined whether certain gait-related parameters correlate with proximal femoral BMD in ambulatory individuals with poststroke walking deficits. We analyzed data from 33 individuals with chronic stroke and found that a recently introduced metric, the Bone Density Index, which incorporates body weight, number of steps per day, and ground reaction force magnitude, predicted proximal femoral BMD better than other commonly measured demographic and gait-related parameters that we examined.
\end{abstract}

Key words: bone mineral density, cerebrovascular accident, gait, gait speed, ground reaction force, osteopenia, osteoporosis, stroke, walking, walking speed.

\section{INTRODUCTION}

The American Heart Association estimates there were 4.8 million stroke survivors in the United States as of 2001 [1]. Among stroke survivors, the ability to walk independently is considered an important functional goal.
While up to 70 percent of stroke survivors recover some ability to walk [1], many have permanent mobility impairments such as decreased walking speed and decreased endurance. Because limitations in mobility often lead to physical inactivity, stroke survivors typically experience a number of disuse-related health issues, such as cardiovascular deconditioning, loss of muscle strength, and osteoporosis.

Researchers have long recognized that stroke survivors experience excess bone loss (i.e., in addition to that

\footnotetext{
Abbreviations: $\mathrm{BDI}=$ Bone Density Index, $\mathrm{BMD}=$ bone mineral density, $\mathrm{BMI}=$ body mass index, $\mathrm{BW}=$ body weight, $\mathrm{DXA}=$ dual-energy X-ray absorptiometry, FIM = Functional Independence Measure, GRF = ground reaction force, MSWS = maximum safe walking speed, ROI = region of interest, SSWS $=$ selfselected walking speed.

This material was based on work supported by the Department of Veterans Affairs, Veterans Health Administration, Rehabilitation Research and Development Service, merit review grant B2748R (Gary S. Beaupre and Steven A. Kautz). *Address all correspondence to Gary S. Beaupre, PhD; Rehabilitation Research and Development Center, MS 153, VA Palo Alto Health Care System, 3801 Miranda Avenue, Palo Alto, CA 94034; 650-493-5000, ext. 64272; fax: 650-493-4919. Email: beaupre@va51.stanford.edu DOI: 10.1682/JRRD.2005.02.0036
} 
associated with normal aging) in the paretic upper limbs and in the lower limbs [2-4]. Bone loss in the lower limb is a particularly serious concern, because stroke survivors have a hip fracture risk two to four times higher than that of age- and sex-matched healthy individuals [5-6]. Hip fracture complicates rehabilitation for the stroke survivor, and the 12-month mortality rate following hip fracture is at least equal to, if not greater than, the mortality rate following hip fracture in nonstroke patients [7-8].

In spite of the serious consequences of osteoporosis, the status, progression, and treatment of this disease in individuals with poststroke hemiparesis have received little attention, especially compared with the long history of research on osteoporosis in healthy postmenopausal women and elderly men. Jørgensen et al. [9] contended that as of 1999 "only three studies ... measured BMD [bone mineral density] in the proximal femur in hemiplegic patients.” More recently, Poole et al. [10] pointed out that "measures to prevent bone loss and preserve bone architecture have not been part of stroke management thus far."

Because hip fracture has such serious health consequences, we believe that bone maintenance in the lower limb should be an important concern of any poststroke rehabilitation plan. Because walking is the most common form of leisure time physical activity, particularly among the elderly [11], regular walking is likely to be critical for bone maintenance at the hip in both healthy and mildly disabled elderly individuals. At present, very little is known about the relationships between walking ability, skeletal loading, and bone maintenance in stroke survivors; thus, what countermeasures might be effective in preventing or modulating post-stroke bone loss in the lower limbs is not clear.

In several recent studies, investigators have examined correlations between bone density or bone loss and various measures of disability and recovery, including the Brunnstrom score [12], Functional Independence Measure (FIM) score [12-13], and Barthel Index [14-15]. Some of these studies showed significant, but modest, correlations, while other studies found none. This lack of consistency provided one of the motivations for the present study. This study, therefore, determines if demographic characteristics (age, weight, body mass index, time since stroke), level of motor recovery (Fugl-Meyer score), and gait-related parameters (walking speed, walking steps per day, ground reaction force [GRF], and the newly developed Bone Density Index [BDI]) are associated with proximal femoral BMD in ambulatory individuals with poststroke walking deficits. Because BMD is strongly linked with skeletal loading [16], we hypothesized that parameters directly related to lower-limb loading history would correlate more strongly with proximal femoral BMD than would other parameters, such as patient demographics, that are not directly loading-related.

\section{METHODS}

Individuals with hemiparesis caused by a single stroke were recruited for this study. Demographics collected for each participant included age, height, weight, BMI, time since stroke, affected side, and use and type of mobility aid (e.g., cane or ankle-foot orthosis). Written informed consent was obtained from all participants, and the Stanford University Administrative Panel on Human Subjects in Medical Research approved the study.

Inclusion criteria were-

- Unilateral weakness.

- Less than 85 years.

- Time since stroke greater than 12 months.

- If female, at least 5 years past the onset of menopause.

- Ability to walk $10 \mathrm{~m}$ in $50 \mathrm{~s}$ or less without contact assistance.

Exclusion criteria were-

- More than one previous cerebral vascular incident.

- Inability to provide informed consent.

- Use of osteoporosis drug or hormone replacement therapy within the past 5 years.

- History of lower-limb fracture or pain.

- Existence of any other medical condition that could affect bone mass.

We scored all participants using the FIM and the Fugl-Meyer assessment tools, which measure disability and recovery, respectively. Both assessment tools have been shown to be valid and reliable [17-19]. Since gaitrelated activity was our only focus, only the FIM locomotion subscore (range 1-7) for walking was evaluated. For the Fugl-Meyer assessment, only the lower-limb performance score (range 0-100) was evaluated.

Each participant's walking was assessed in our gait laboratory. Self-selected walking speed (SSWS) and maximum safe walking speed (MSWS) were measured while each participant walked on a 4.3 m-long GAITRite portable walkway system (CIR Systems, Inc, Clifton, New Jersey). Additionally, we measured the three-dimensional 
components of the GRF throughout the stance phase for both the paretic and nonparetic sides. The GRF was measured while each participant walked at his or her SSWS along a $10 \mathrm{~m}$ walkway equipped with embedded force platforms (Advanced Medical Technology, Inc, Watertown, Massachusetts; Bertec Corporation, Columbus, Ohio). GRF data were acquired at $200 \mathrm{~Hz}$, and the vertical GRF component (normalized by each individual's body weight) during the support phase for each limb was used for analysis. We averaged a minimum of 2 trials and a maximum of 15 trials to determine gait speed and GRF for each participant.

So that we could measure gait activity outside of the laboratory setting, we provided individuals with a StepWatch 2 Activity Monitor (Cyma Corporation, Seattle, Washington) and gave instruction in its use [20-21]. The StepWatch records the number of steps taken for one leg during walking, including level, uphill, and downhill walking, as well as during stair ascent and descent. The device requires no input on the part of the user, except for attaching the device upon rising each morning and removing the device at bedtime each evening. The device was not worn during bathing. The StepWatch device was set at the company-recommended settings for individuals with expected slow gait speeds.

We measured BMD in the total femur region of interest (ROI) on both the paretic and nonparetic sides using dual-energy X-ray absorptiometry (DXA) (QDR 1000W, Hologic Inc, Bedford, Massachusetts). Participants were categorized as normal, osteopenic, or osteoporotic based on their DXA T-scores. The classification is based on the World Health Organization criteria, with normal defined as a T-score of greater than or equal to (i.e., more positive) -1.0 , osteopenic defined as a T-score of less than -1.0 and greater than -2.5 , and osteoporotic defined as a T-score of less than or equal to -2.5 [22]. The total femur ROI was chosen based on recommendations that it is the preferred ROI for densitometry measurements in the appendicular skeleton [23-24].

In addition to the traditional gait parameters described previously, we also calculated a BDI, as developed by Bowley and Whalen [25]. The BDI is calculated as

$$
\mathrm{BDI}=\left(n_{\text {steps }} \times(\beta \times \mathrm{GRF})^{m}\right)^{1 /(2 m)},
$$

where $n_{\text {steps }}$ is the number of steps taken by each leg per day obtained from the StepWatch, $\beta$ is a scale factor obtained by dividing each individual's weight by the mean body weight (BW) of the entire cohort, GRF is either the peak or mean value of the vertical component of the GRF during BW support [26], and $m$ is an empirical exponent that can be thought of as weighting factor for the relative importance of load magnitude and the number of daily loading cycles [27-28]. We tested integer values of $m$ between 1 and 6 to determine which value produced the highest value of the correlation coefficient between BDI and total proximal femur BMD.

Statistical comparisons between paretic and nonparetic sides are based on paired two-tailed $t$-tests with the level of significance set at $p=0.05$.

\section{RESULTS}

The study included 33 individuals (29 male, 4 female). Table 1 shows the characteristics of the study participants. Of the 33 participants, 8 individuals used either a straight or quad cane, 8 used a plastic ankle-foot orthosis, and 2 used a foot splint. Fifteen participants had left-side lesions, and eighteen had right-side lesions. Because our inclusion criteria required participants to be able to walk with no contact assistance, all participants had an FIM locomotion score of at least 5 .

Table 2 shows the summary results of the walking speed tests and daily StepWatch totals. Two individuals were unable to increase their walking speed above their SSWS; in one, the limitation was ankle spasms and in the other the limitation was stumbling and an unsafe gait. Therefore, values given for the MSWSs are based on 31 participants. Using the StepWatch device, we recorded an average of 13 days of step counts. The fewest number of days recorded was 5 for one participant. The next fewest was 9 day for two participants.

Table 1.

Demographic characteristics of study participants.

\begin{tabular}{lcc}
\hline \multicolumn{1}{c}{ Participant Characteristics } & Mean \pm SD & Range \\
\hline Age $(\mathrm{yr})$ & $65 \pm 8$ & $46-81$ \\
Height $(\mathrm{m})$ & $1.76 \pm 0.008$ & $1.55-1.91$ \\
Weight $(\mathrm{kg})$ & $87.3 \pm 14.3$ & $64.8-119.0$ \\
BMI $\left(\mathrm{kg} / \mathrm{m}^{2}\right)$ & $28.2 \pm 3.5$ & $21.5-35.2$ \\
Time Since Stroke $(\mathrm{mo})$ & $45.9 \pm 29.1$ & $12-121$ \\
FIM Locomotion Score (max $=7)$ & $5.9 \pm 0.6$ & $5-7$ \\
Fugl-Meyer Lower-Limb Score $(\max =100)$ & $86 \pm 8$ & $63-98$ \\
\hline
\end{tabular}

$\mathrm{SD}$ = standard deviation, BMI = body mass index, FIM = Functional Independence Measure. 
Table 3 shows the results of linear correlation analysis between total proximal femur BMD and various demographic and gait parameters. Shown are the correlation coefficients $(r)$ and the significance values $(p)$. After calculating the BDI values for $m$ values of $1-6$, we found that an $m$ value of 3 resulted in the best fit when correlated with total proximal femur BMD. All reported values for the BDI are based on $m=3$.

We found no significant correlations between BMD and age, weight, BMI, time since stroke, Fugl-Meyer score, SSWS, MSWS, or peak GRF for either the paretic or nonparetic sides. We found a significant correlation between BMD and steps per day for the paretic side but not for the nonparetic side. We found a significant correlation between BMD and mean GRF for the nonparetic side, but not for the paretic side. For both the paretic and nonparetic sides, we found that BMD significantly correlated with two parameters: BDI calculated using peak GRF and BDI calculated using mean GRF values. The strongest correlations were between BMD and BDI based on the mean GRF, with correlation coefficients of 0.418 and 0.473 for the paretic and nonparetic sides, respectively. Linear regressions between BMD and BDI based on mean GRF (BDI_m) are shown in the Figure. Table 4 shows summary data (mean \pm standard deviation and range) for the paretic and nonparetic sides for BMD, T-score, mean and peak GRF, and BDI values based on mean and peak GRF.

Based on the individual T-scores for the total femur ROI (not shown), 17 participants were in the normal range, 13 were osteopenic, and 3 were osteoporotic. We found significant side-to-side differences for total proximal femur BMD, total proximal femur T-score, mean GRF, and BDI based on the mean GRF. Peak GRF and BDI based on peak GRF were not different between sides. Note that the values $(0.90 \mathrm{BW})$ at the low end of the range for the peak GRF correspond to individuals who walked with a cane.

Table 2.

Group walking characteristics of study participants.

\begin{tabular}{lcc}
\hline \multicolumn{1}{c}{ Gait-Related Variables } & Mean \pm SD & Range \\
\hline Self-Selected Walking Speed $(\mathrm{m} / \mathrm{s})$ & $0.79 \pm 0.34$ & $0.23-1.34$ \\
$\begin{array}{l}\text { Maximum Safe Walking Speed } \\
(\mathrm{m} / \mathrm{s})(n=31)\end{array}$ & $1.18 \pm 0.49$ & $0.37-2.13$ \\
Steps Per Leg Per Day & $2,971 \pm 1,449$ & $1,013-6,564$ \\
\hline SD = standard deviation. & & \\
\hline \hline
\end{tabular}

Table 3.

Correlation coefficients $(r)$ and significance values $(p)$ for demographic and gait-related parameters (at self-selected speed) versus bone mineral density (BMD). Ground reaction force (GRF) and Bone Density Index (BDI) values are based on self-selected walking speed (SSWS) data. Parameters in bold indicate significant correlations $(p<0.05)$.

\section{Demographic and Gait-Related Parameters}

Total Proximal Femur BMD $\left(\mathrm{g} / \mathrm{cm}^{2}\right)$ Correlation Coefficients and Significance Levels

\begin{tabular}{lcccc}
\multicolumn{1}{c}{ Parameters } & \multicolumn{2}{c}{ Paretic } & \multicolumn{2}{c}{ Nonparetic } \\
\cline { 2 - 4 } \cline { 3 - 5 } & \multicolumn{1}{c}{$\boldsymbol{r}$} & $\boldsymbol{p}$-Value & $\boldsymbol{r}$ & $\boldsymbol{p}$-Value \\
\hline Age (yr) & 0.050 & 0.784 & 0.294 & 0.097 \\
Weight (kg) & 0.118 & 0.514 & 0.257 & 0.150 \\
BMI (kg/m ${ }^{2}$ ) & 0.060 & 0.739 & 0.067 & 0.712 \\
Time Since Stroke (mo) & 0.057 & 0.750 & 0.090 & 0.619 \\
Fugl-Meyer score & 0.197 & 0.271 & 0.084 & 0.642 \\
SSWS (m/s) & 0.178 & 0.321 & 0.191 & 0.287 \\
MSWS (m/s) (n=31) & 0.182 & 0.311 & 0.229 & 0.227 \\
GRF Peak (BW) & 0.280 & 0.115 & 0.158 & 0.378 \\
Steps Per Leg Per Day & $\mathbf{0 . 3 7 6}$ & $\mathbf{0 . 0 3 1}$ & 0.253 & 0.156 \\
GRF Mean (BW) & 0.333 & 0.058 & $\mathbf{0 . 3 9 3}$ & $\mathbf{0 . 0 2 4}$ \\
BDI_p (based on peak GRF) & $\mathbf{0 . 3 9 9}$ & $\mathbf{0 . 0 2 2}$ & $\mathbf{0 . 3 9 9}$ & $\mathbf{0 . 0 2 2}$ \\
BDI_m (based on mean GRF) & $\mathbf{0 . 4 1 8}$ & $\mathbf{0 . 0 1 5}$ & $\mathbf{0 . 4 7 3}$ & $\mathbf{0 . 0 0 5}$ \\
\hline B
\end{tabular}



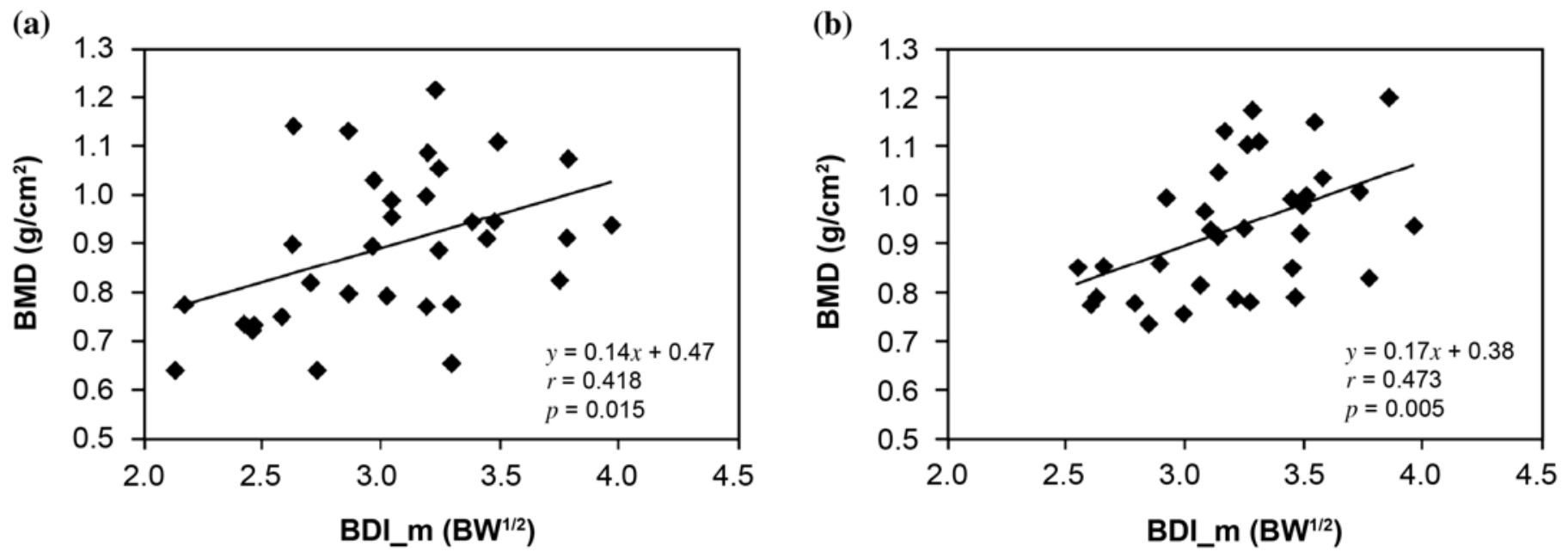

Figure.

Relationship between bone mineral density (BMD) for the total femur region of interest and Bone Density Index (BDI) based on mean GRF (BDI_m) for (a) paretic and (b) nonparetic sides. Regression equations, correlation coefficients $(r)$, and $p$-values are reported. BW = body weight.

Table 4.

Values for bone mineral density (BMD), T-score, and gait-related parameters for paretic and nonparetic sides (mean \pm standard deviation and range. Ground reaction force (GRF) and Bone Density Index (BDI) values are based on self-selected walking speed data. Parameters in bold indicate significant side-to-side differences $(p<0.05)$.

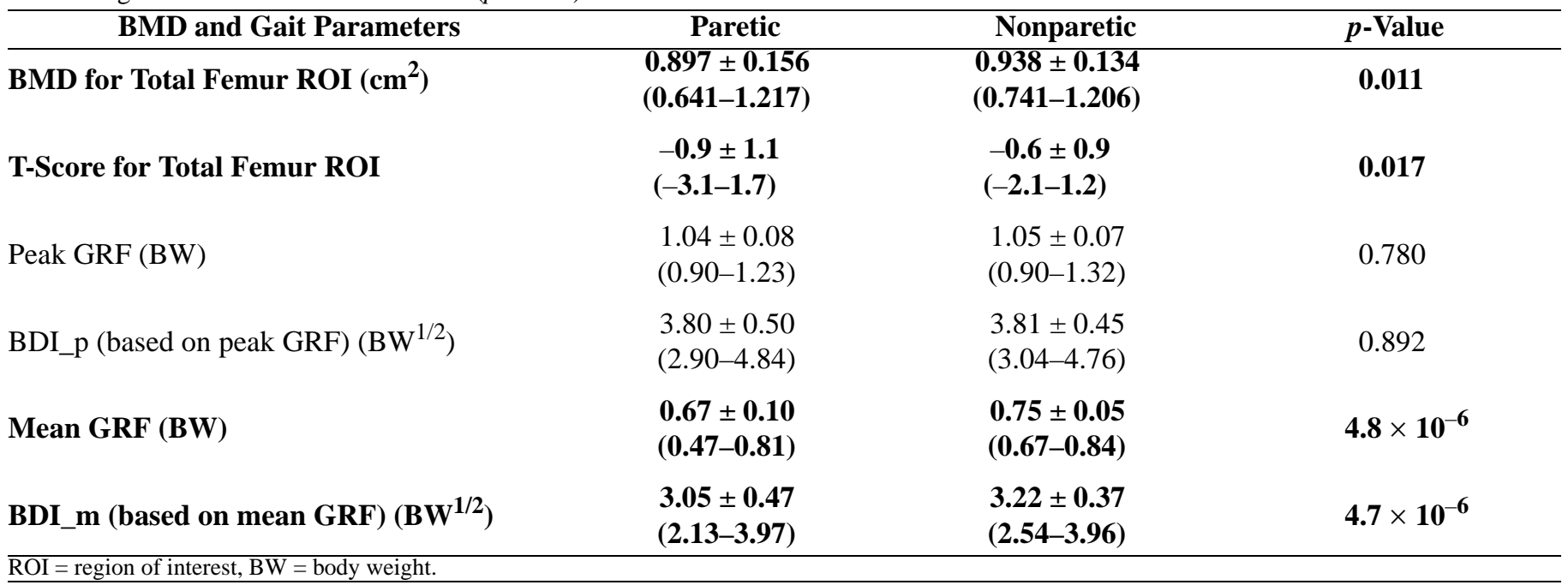

\section{DISCUSSION}

In agreement with previous studies, we found a lower proximal femoral BMD on the paretic side compared with the nonparetic side [29-31]. We found that this decreased BMD was not correlated with demographic or clinical motor recovery variables. We found significant correlations between BMD and the newly developed gait-related parameter, the BDI. The strongest correlations were found when the BDI was based on the mean vertical GRF experienced during walking. While we defined the BDI based on the vertical component of the GRF to be consistent with the initial work of Bowley and Whalen [25], we also reasonably based the definition of the BDI on the vector resultant of the vertical, fore-aft, and medial-lateral GRF components, and this idea is worthy of further study. In any case, our current results support the hypothesis that a parameter that includes information about the daily skeletal 
loading history will correlate better with BMD than will other commonly measured demographic and gait-related parameters.

Our results suggest that increasing the magnitude of the vertical GRF during walking should be beneficial for poststroke BMD maintenance. This result is consistent with several studies that focused on the importance of the relationship between GRF and BMD at weight-bearing skeletal sites in neurologically unimpaired populations [32-34].

The BDI developed by Bowley and Whalen [25] was chosen because their theoretical framework addressed our hypothesis and because the measure was relatively simple to adapt for use with stroke survivors. We adapted the BDI for use with the group we studied in several ways. Bowley and Whalen used a more complicated definition of the BDI, in which they used a histogram of vertical GRF values and associated step counts obtained from a special load-sensing insole that was capable of recording the step-by-step peak vertical GRF values for each volunteer over the course of multiple days. We instead measured GRF in stroke survivors by using force platforms embedded in the laboratory floor and assumed that data recorded from 2 or more steps of walking at their SSWS were representative of forces experienced during daily habitual walking. That the BDI correlation coefficients for our cohort are so similar to the correlation coefficient $(0.437)$ found by Bowley and Whalen may indicate that for studies involving subjects whose primary locomotion activity is slow walking, monitoring the GRF values continuously as was done by Bowley and Whalen may not be necessary. For these individuals, their laboratory performance may represent the majority of their normal locomotor activity.

The results of this study have implications for the use of gait training as an osteoporosis countermeasure. In particular, the role of increased vertical GRFs in BMD maintenance is promising as a therapeutic intervention. In this study, as in previous studies, the empirical exponent term $(m)$ in the bone-remodeling algorithm was found to be larger than $1.0[25,27]$. This implies that a given percentage increase in the force parameter of the BDI will have a larger effect on BMD than the same percentage increase in the number of loading cycles. The relationship between GRF and gait speed has been documented in individuals without neurological disorders [35]. For speeds greater than approximately $0.8 \mathrm{~m} / \mathrm{s}$, dynamic factors influence forces such that increased speed causes peak vertical GRF in excess of 1.0 BW. Perry et al. have suggested that increasing gait speed should be an important outcome measure for poststroke rehabilitation programs [36]. Perry et al. found that $0.8 \mathrm{~m} / \mathrm{s}$ is the mean gait speed of the highest category of community ambulators in a study of 147 stroke survivors, and these authors therefore suggest that a threshold of $0.8 \mathrm{~m} / \mathrm{s}$ should be a goal in gait rehabilitation for functional independence in a community setting. In combination with our study, these results suggest multiple important benefits that patients would accrue if a locomotor rehabilitation program were able to safely increase their gait speed. Our results suggest that stroke survivors able to maintain the number of steps taken per day while increasing walking speed and associated vertical GRF applied during each step would experience benefits in lower-limb skeletal health. These results highlight the importance of identifying locomotor rehabilitation programs that might help attain gait speeds high enough to generate functional independence and BMD benefits. Clearly, increasing walking speed as an osteoporosis countermeasure in stroke survivors is only a hypothetical possibility at present, and the potential of such an approach requires further study.

Although our study was open to both men and women, only 4 of the 33 participants were women. Current or recent hormone replacement therapy and osteoporosis drug treatment, which would potentially confound our results because of their known influence on $\mathrm{BMD}$, were the most common reasons for excluding some women. Because of the small number of women who participated, we were not able to test whether men and women have different correlations between BMD and any of the various gait parameters examined, but we have no reason to believe that daily mechanical loading is any less important for women.

Finally, we can speculate that proximal femur BMD may be more closely regulated by a local, rather than distant, measure of loading. In a future study we plan to estimate subject-specific hip-joint reaction forces by simulating stroke gait using kinematic and kinetic data from motion analysis to determine if the BDI based on the cyclic hip-joint reaction force produces a stronger correlation with proximal femur BMD than does BDI based on GRF.

\section{CONCLUSIONS}

In a cohort of 33 ambulatory individuals with chronic stroke, we found that the newly introduced BDI, which 
incorporates both loading magnitude and the number of daily loading cycles correlated significantly with proximal femur BMD. Our results suggest that (1) the BDI may be a useful parameter for predicting bone density in ambulatory stroke survivors and (2) increasing daily walking activity and increasing GRFs may potentially modulate bone loss and should therefore be considered when devising stroke rehabilitation protocols for those stroke survivors who are independent ambulators.

\section{ACKNOWLEDGMENTS}

A special thanks to Dr. Jill Higginson for help in ensuring gait data quality, Barbara Elspas for assistance with participant recruiting, and Nancy Wei for assistance with DXA scanning.

\section{REFERENCES}

1. American Heart Association. Heart disease and stroke statistics—2004 update. Dallas (TX): American Heart Association; 2003.

2. Peszczynski M. The fractured hip in hemiplegic patients. Geriatrics. 1957;12(12):687-90.

3. Mulley G, Espley AJ. Hip fracture after hemiplegia. Postgrad Med J. 1979;55(642):264-65.

4. Prince RL, Price RI, Ho S. Forearm bone loss in hemiplegia: A model for the study of immobilization osteoporosis. J Bone Miner Res. 1988;3(3):305-10.

5. Ramnemark A, Nyberg L, Borssen B, Olsson T, Gustafson Y. Fractures after stroke. Osteoporos Int. 1998;8(1):92-95.

6. Kanis J, Oden A, Johnell O. Acute and long-term increase in fracture risk after hospitalization for stroke. Stroke. 2001;32(3):702-6.

7. Youm T, Aharonoff G, Zuckerman JD, Koval KJ. Effect of previous cerebrovascular accident on outcome after hip fracture. J Orthop Trauma. 2000;14(5):329-34.

8. Ramnemark A, Nilsson M, Borssen B, Gustafson Y. Stroke, a major and increasing risk factor for femoral neck fracture. Stroke. 2000;31(7):1572-77.

9. Jørgensen L, Jacobsen BK, Wilsgaard T, Magnus JH. Walking after stroke: Does it matter? Changes in bone mineral density within the first 12 months after stroke. A longitudinal study. Osteoporos Int. 2000;11(5):381-87.

10. Poole KE, Reeve J, Warburton EA. Falls, fractures, and osteoporosis after stroke: Time to think about protection? Stroke. 2002;33(5):1432-36.

11. Centers for Disease Control and Prevention (CDC). Compliance with physical activity recommendations by walking for exercise-Michigan, 1996 and 1998. MMWR Morb Mortal Wkly Rep. 2000;49(25):560-65.

12. Yavuzer G, Ataman S, Suldur N, Atay M. Bone mineral density in patients with stroke. Int J Rehabil Res. 2002; 25(3):235-39.

13. Liu M, Tsuji T, Higuchi Y, Domen K, Tsujiuchi K, Chino $\mathrm{N}$. Osteoporosis in hemiplegic stroke patients as studied with dual-energy X-ray absorptiometry. Arch Phys Med Rehabil. 1999;80(10):1219-26.

14. Sahin L, Ozoran K, Gunduz OH, Ucan H, Yucel M. Bone mineral density in patients with stroke. Am J Phys Med Rehabil. 2001;80(8):592-96.

15. Watanabe Y. An assessment of osteoporosis in stroke patients on rehabilitation admission. Int J Rehabil Res. 2004; 27(2):163-66.

16. Carter DR, Beaupre GS. Skeletal function and form: Mechanobiology of skeletal development, aging, and regeneration. New York (NY): Cambridge University Press; 2001.

17. Fugl-Meyer AR, Jaasko L, Leyman I, Olsson S, Steglind S. The post-stroke hemiplegic patient. 1. A method for evaluation of physical performance. Scand J Rehabil Med. 1975; 7(1):13-31.

18. Duncan PW, Propst M, Nelson SG. Reliability of the FuglMeyer assessment of sensorimotor recovery following cerebrovascular accident. Phys Ther. 1983;63(10):1606-10.

19. Hamilton BB, Laughlin JA, Fiedler RC, Granger CV. Interrater reliability of the 7-level functional independence measure (FIM). Scand J Rehabil Med. 1994;26(3):115-19.

20. Coleman KL, Smith DG, Boone DA, Joseph AW, Del Aguila MA. Step activity monitor: Long-term, continuous recording of ambulatory function. J Rehabil Res Dev. 1999; 36(1):8-18.

21. Resnick B, Nahm ES, Orwig D, Zimmerman SS, Magaziner J. Measurement of activity in older adults: Reliability and validity of the Step Activity Monitor. J Nurs Meas. 2001;9(3):275-90.

22. Assessment of fracture risk and its application to screening for postmenopausal osteoporosis. Report of a WHO study group. World Health Organ Tech Rep Ser. 1994;843:1-129.

23. Centers for Disease Control and Prevention (CDC). Osteoporosis among estrogen-deficient women-United States, 1988-1994. MMWR Morb Mortal Wkly Rep. 1998; 47(45):969-73.

24. Fogelman I, Blake GM. Different approaches to bone densitometry. J Nucl Med. 2000;41(12):2015-25.

25. Bowley SM, Whalen RT. Physical activity and bone density in women. Trans Orthop Res Soc. 2001;26:63.

26. Kim CM, Eng JJ. Magnitude and pattern of 3D kinematic and kinetic gait profiles in persons with stroke: Relationship to walking speed. Gait Posture. 2004;20(2):140-46. 
27. Whalen RT, Carter DR, Steele CR. Influence of physical activity on the regulation of bone density. J Biomech. 1988; 21(10):825-37.

28. Beaupre GS, Orr TE, Carter DR. An approach for timedependent bone modeling and remodeling-Theoretical development. J Orthop Res. 1990;8(5):651-61.

29. Del Puente A, Pappone N, Mandes MG, Mantova D, Scarpa R, Oriente P. Determinants of bone mineral density in immobilization: A study on hemiplegic patients. Osteoporos Int. 1996;6(1):50-54.

30. Takamoto S, Masuyama T, Nakajima M, Seikiya K, Kosaka H, Morimoto S, Ogihara T, Onishi T. Alterations of bone mineral density of the femurs in hemiplegia. Calcif Tissue Int. 1995;56(4):259-62.

31. Ramnemark A, Nyberg L, Lorentzon R, Englund U, Gustafson Y. Progressive hemiosteoporosis on the paretic side and increased bone mineral density in the nonparetic arm the first year after severe stroke. Osteoporos Int. 1999;9(3):269-75.
32. Welsh L, Rutherford OM. Hip bone mineral density is improved by high-impact aerobic exercise in postmenopausal women and men over 50 years. Eur J Appl Physiol Occup Physiol. 1996;74(6):511-17.

33. Snow CM, Shaw JM, Winters KM, Witzke KA. Long-term exercise using weighted vests prevents hip bone loss in postmenopausal women. J Gerontol A Biol Sci Med Sci. 2000;55(9):M489-91.

34. Jessup JV, Horne C, Vishen RK, Wheeler D. Effects of exercise on bone density, balance, and self-efficacy in older women. Biol Res Nurs. 2003;4(3):171-80.

35. Andriacchi TP, Ogle JA, Galante JO. Walking speed as a basis for normal and abnormal gait measurements. J Biomech. 1977;10(4):261-68.

36. Perry J, Garrett M, Gronley JK, Mulroy SJ. Classification of walking handicap in the stroke population. Stroke. 1995; 26(6):982-89.

Submitted for publication February 4, 2005. Accepted in revised form June 29, 2005. 